This document is confidential and is proprietary to the American Chemical Society and its authors. Do not copy or disclose without written permission. If you have received this item in error, notify the sender and delete all copies.

\title{
Strain Dependent Dielectric Behavior of Carbon Black Reinforced Natural Rubber
}

\begin{tabular}{|r|l|}
\hline Journal: & Macromolecules \\
\hline Manuscript ID & Draft \\
\hline Manuscript Type: & Article \\
\hline Domplete List of Authors: & $\begin{array}{l}\text { HUANG, MENGLONG; Queen Mary University of London, School of } \\
\text { Engineering and Materials Science } \\
\text { Tunnicliffe, Lewis; Queen Mary University of London, School of Engineering } \\
\text { and Materials Science } \\
\text { Zhuang, Jian; Xi'an Jiaotong University, Electronic Materials Research } \\
\text { Laboratory, Key Laboratory of the Ministry of Education } \\
\text { Ren, Wei; Xi'an Jiaotong University, Electronic Materials Research } \\
\text { Laboratory, Key Laboratory of the Ministry of Education } \\
\text { Yan, Haixue; Queen Mary, University of London, School of Engineering and } \\
\text { Materials Science } \\
\text { Busfield, J.; Queen Mary University of London, School of Engineering and } \\
\text { Materials Science }\end{array}$ \\
\hline & \begin{tabular}{l} 
\\
\hline
\end{tabular} \\
\hline
\end{tabular}

SCHOLARONE ${ }^{\text {m }}$

Manuscripts 


\title{
Strain Dependent Dielectric Behavior of
}

\section{Carbon Black Reinforced Natural Rubber}

\author{
Menglong Huang, ${ }^{\dagger, t}$ Lewis B. Tunnicliffe, ${ }^{\dagger, t}$ Jian Zhuang, ${ }^{\S}$ Wei Ren ${ }^{\S}$ Haixue Yan, ${ }^{\dagger, t}$ \\ James J.C. Busfield ${ }^{*}+$, t \\ ${ }^{\dagger}$ School of Engineering and Materials Science, Queen Mary University of London, \\ Mile End Road, London, E1 4NS, UK \\ ${ }^{\ddagger}$ Materials Research Institute, Queen Mary University of London, Mile End Road, \\ London, E1 4NS, UK \\ ${ }^{\S}$ Electronic Materials Research Laboratory, Key Laboratory of the Ministry of \\ Education \& International Centre for Dielectric Research, Xi'an Jiaotong University, \\ Xi'an 710049, China
}

KEYWORDS: Nanodielectric, carbon black, natural rubber, permittivity, dipole, interface

\begin{abstract}
The nature of filler-polymer and filler-filler interactions in rubber composites under strain remains an open question in soft matter physics. These interactions are key to explaining the rich variety of complex behavior exhibited by particle-filled rubber products. In this paper we demonstrate a simultaneous dielectric/dynamic mechanical analysis technique (SDMS) which provides new insights into the structure-property relationships of filled rubbers. The complex
\end{abstract}


permittivity of carbon black filled natural rubber has been characterized under a simultaneous tensile strain field (from $0.1 \%$ to $50 \%$ ). The complex permittivity exhibits a dramatic non-linear dependence on strain coupled with features which are analogous to mechanical strain softening and strain history namely the 'Payne' and 'Mullins' effects. The sensitivity of the complex permittivity to such effects is several orders of magnitude greater than in corresponding, traditional mechanical tests. In addition, we demonstrate for the first time that it is possible to use both strain and electrical field frequency as 'dipole filters' which can be used to selectively probe the dipoles present at the polymer-filler interface.

\section{INTRODUCTION}

Nano-structured particulate fillers such as carbon black and precipitated silica are widely used as the primary reinforcing materials in the tire and rubber industries. Such fillers are often used to provide modifications to the static and dynamic moduli, fracture and abrasion resistance and, more generally, as a means to modify the strain dependent viscoelastic spectrum of rubber materials. However, although such technology has been in use for well over a century, the precise physical mechanisms behind the reinforcement phenomenon are still not clearly understood or agreed upon.

Over the past century a wide range of predominantly mechanical-based experimental techniques have been used to investigate the mechanisms of reinforcement. ${ }^{1-3}$ Only relatively recently has significant, focused attention been paid to the characterization and interpretation of the dielectric properties of reinforced rubbers. Dielectric investigations present new opportunities for furthering the understanding of reinforcement based upon the sensitivity of the technique to 
observing changes in the morphology of flocculated or jammed filler particle networks as well as the interactions between filler particles and the polymer matrix. Thus, another possible novel approach to understanding the mechanical behavior of filled rubbers under mechanical deformation is to simultaneously evaluate their mechanical and dielectric properties. Throughout this paper, the dielectric properties are presented as the complex permittivity (simplified as permittivity in the following parts): $\kappa^{*}=\kappa^{\prime}-i \kappa^{\prime \prime}$ where $\kappa^{\prime}$ and $\kappa^{\prime \prime}$ are the real and imaginary part of permittivity respectively. The dielectric loss is defined as $D=\kappa^{\prime \prime} / \kappa^{\prime}$.

Elastomers filled with nano-particles have also shown great potential in energy harvesting, ${ }^{4}$ actuating ${ }^{5}$ and sensor devices ${ }^{6}$ due to their relatively low moduli and optimized dielectric and mechanical properties. For such applications $\kappa^{*}$ is a critical parameter. However, in the literature we find that in most of the cases, $\kappa^{*}$ is measured and discussed under static (non-deformed) conditions and is treated as a constant value during the devices' working life - despite the fact that such devices would normally operate in a dynamic mechanical environment. The dielectric performance of such materials in the dynamically deformed state is rarely discussed.

\subsection{Mechanical Properties of Reinforced Rubbers. In rubber science and} technology, the mechanical properties of filler-reinforced rubbers ${ }^{3}$ are intimately linked to the filler particle size and morphology, the strain dependence of the flocculated/jammed filler network and the filler-polymer interaction(s). Key phenomenology associated with filler reinforcement is the onset of a non-linear strain dependence of the viscoelastic properties with increasing filler volume fraction, $\phi$ (called the Payne effect). ${ }^{7}$ Additionally, particle reinforced rubbers can also exhibit significant strain history effects at finite deformations (Mullins effect) ${ }^{8}$ and cyclic stress softening phenomena. A range of microstructural mechanisms have been 
proposed to account for this behavior including rupturing of the filler network, ${ }^{7,9}$ polymer-filler interfacial debonding, ${ }^{10}$ the strain dependence of interphase polymer ${ }^{11}$ and the unjamming of the filler network through the application of mechanical and/or thermal energy. ${ }^{12,13}$ However, none of these mechanisms currently provide a unified microstructural description of the reinforcement phenomenon.

1.2. Dielectric Properties of Reinforced Rubbers. The permittivity of heterogeneous systems such as filled composites, polymer blends, or porous materials have been discussed for over a century. A number of different approaches to understanding mixture laws and models for such systems have been reported ${ }^{14,15}$ since initial work by Maxwell. Lewis ${ }^{16}$ claimed that the interface plays the most important role in multi-phase systems. Following this work, a number of theoretical models such as Tanaka's multi-core mode ${ }^{17}$ have been proposed to describe the physics of the interface. However, the electrical structure of the interface is still not clearly understood and the literature suffers from a lack of detailed experimental studies.

The bulk permittivity of any material is composed of contributions from a range of different dipoles of varying length and time scales. For the case of particle-reinforced rubbers, certain dipoles, localized in different parts of the composite, are of particular significance. Firstly, polarization of polymer chains results in dipoles which can be classified by the dipole orientation. ${ }^{18}$ For example, A-type dipoles, which are aligned parallel to the main chain, reveal the global motion of the whole chain. At smaller length and shorter time scales, dipoles aligned perpendicular to the main chain reflect the chain segmental motions underpinning the polymer glass transition, and are called B-type. Secondly, it has been proposed that polymer in close proximity to the filler surface may exhibit a gradient of slower dynamics compared with that of the bulk, 
unfilled polymer. ${ }^{19}$ Such interphase material has been reported to have raised glass transition temperature $\left(T_{g}\right)$ to such an extent that 'glassy layers' of several nanometers thick surrounding filler particles have been claimed to dominate the bulk mechanical and dielectric properties of filled rubbers. ${ }^{20-22}$ Therefore, the dipoles in such interphase polymer may behave differently in comparison to the bulk matrix. However, there is an active debate as to the existence of 'glassy layers' and their applicability to commercial materials. Such interphase polymer is found to be highly dependent on the filler size and dispersion which clearly varies from case to case. ${ }^{21,23,}$ 24 Thirdly, charge transfer between filler-polymer interfaces can generate surface dipoles and form an electrical double layer. ${ }^{16,25,26}$ This charge transfer or charge distribution may come from the differences in the degree of alignment of Femi levels of the components but highly depends on the type of contact. ${ }^{25}$ Moreover this behavior becomes rather complex as recently it has been shown to also depend on mechanical strain. ${ }^{27}$ Fourthly, charges (space charges) will gather on the interface when the current density varies from one phase to another phase. This is known as interfacial polarization or Maxwell-Wagner-Sillars (MWS) polarization. ${ }^{28-30}$ It is dominant at low electrical frequencies $(f)$ and becomes weaker with increasing $f$. Finally, dipoles located inside the fillers themselves can also contribute to the measured $\kappa^{*}$. For clarity, we assign each of the dipoles mentioned above the following names: BULK-dipole, GLASSY-dipole, CHARGE-dipole, MWS-dipole, and FILLER-dipole respectively.

Over the past few decades, dielectric spectroscopy has been used to study filler particle flocculation ${ }^{31}$ and distribution, ${ }^{32}$ filler network structure, ${ }^{33}$ strain induced crystallization $^{34}$ and compound degradation. ${ }^{35}$ These investigations have demonstrated that the dielectric method is highly sensitive to the filler structure. However, only a 
few of these investigations ${ }^{33,34,36-38}$ have applied the dielectric method to deformed rubber materials. Furthermore, none of these studies have reported simultaneous insitu measurements. In this way their results are strongly affected by the time dependent viscoelastic nature of the polymer matrix. This may be due, in part, to fact that traditional dielectric spectroscopy uses fixed, rigid electrodes to ensure a good connection between the electrode and the sample.

This article reports on an innovative experimental technique which combines an impedance meter (LCR meter) and a dynamic mechanical analyzer (DMA) in order to accurately characterize the dielectric properties of carbon black N330 (CB) reinforced natural rubber (NR) simultaneously with the application of strain (from around $0.1 \%$ up to $50 \%$ ). In practice this is achieved using compliant silver nanowire (AgNW) electrodes which are deformable and highly conductive under strain. ${ }^{39}$ Although this idea has been experimentally adopted in applications such as force sensing, ${ }^{40}$ they have previously not been used to measure the dielectric properties and microstructural evolution of elastomer composites under a simultaneous small strain. We find that such simultaneous dielectric-mechanical spectroscopy (SDMS) can provide detailed information about the contributions of various dipoles to bulk dielectric behavior and their frequency $(f)$ and strain $(e)$ dependence. This technique offers a new way to complement typical mechanical spectroscopy measurements.

\section{EXPERIMENTAL SECTION}

2.1. Materials. Natural rubber (SMR CV60)/carbon black (HAF N330) masterbatch $(\phi=0.204)$ was prepared by compounding in a Banbury-type internal mixer for 5 minutes at around $149^{\circ} \mathrm{C}$ with a rotor speed of $80 \mathrm{rpm}$ (performed at Cabot Corporation, Boston, USA). To obtain a range of volume fractions 
(summarized in Table 1), the masterbatch was suitably diluted by compounding with unfilled NR on a 2-roll mill. At the same time, dicumyl peroxide (Sigma Aldrich) curative was compounded in at a NR:DCP w/w ratio of 50:1. Samples were then cured at $160{ }^{\circ} \mathrm{C}$ under a pressure of around 60 bars for 1 hour. Each sample has a thickness of around $0.7 \mathrm{~mm}$.

Table 1. Samples codes and corresponding volume fractions used in this study

\begin{tabular}{c|c}
\hline SAMPLE CODE & $\begin{array}{c}\text { VOLUME FRACTION OF } \\
\text { N330 CARBON BLACK }\end{array}$ \\
\hline $\mathbf{N R} / \mathbf{0}$ & 0.000 \\
$\mathbf{N R} / \mathbf{0 . 0 2 5}$ & 0.025 \\
$\mathbf{N R} / \mathbf{0 . 0 4 9}$ & 0.049 \\
$\mathbf{N R} / \mathbf{0 . 0 7 1}$ & 0.071 \\
$\mathbf{N R} / \mathbf{0 . 0 9 3}$ & 0.093 \\
$\mathbf{N R} / \mathbf{0 . 1 1}$ & 0.113 \\
$\mathbf{N R / 0 . 1 3}$ & 0.133 \\
$\mathbf{N R} / \mathbf{0 . 1 5}$ & 0.152 \\
$\mathbf{N R / 0 . 1 9}$ & 0.187 \\
\hline
\end{tabular}

2.2. Tensile Mechanical Tests. Samples were cut into dumbbell shapes for tensile tests according to ASTM D 412. Tests were all performed on an Instron 5566 tensile machine with a $1 \mathrm{kN}$ load cell and a video extensometer. The strain rate was around $17 \% / \mathrm{s}$

2.3. Electrical Test (dc). Keithley 6517A electrometer was used to measure the dc conductivity of all the samples via unshielded connection method. The dimension of each sample was $20 \mathrm{~mm} \times 20 \mathrm{~mm} \times 0.7 \mathrm{~mm}$. A pair of copper disc electrodes with a diameter of $14 \mathrm{~mm}$ were used with the sample was sandwiched in between. A voltage 
potential was applied across the thickness of the sample and the current was measured from which the resistance was calculated using Ohm's law. Finally, the conductivity was obtained based on the sample geometry.

2.4. Dielectric Test without Strain. (A). $0.1 \mathrm{~Hz}-1 \mathrm{MHz}$ was measured by Novocontrol Concept 40 Impedance Spectrometer System with a disk plane capacitor cell at room temperature. In this study this technique was used as a standard dielectric test. The surfaces of each specimens were coated by gold using vacuum deposition in order to ensure a good contact with the electrodes. (B). 7-40 GHz was measured via transmission/reflection technique with the help of a Walton 37269A Network Analyzer at room temperature. Specimens were cut to perfectly fit in different wave guides depending on the testing electrical frequency. The details and theory relating to this technique can be found in the literature. ${ }^{41}$

\subsection{Simultaneous Dielectric-Mechanical Spectroscopy (SDMS). (A). Electrodes} preparation: The raw AgNW-isopropanol suspension (Blue Nano Inc., USA) was diluted to $0.0025 \mathrm{mg} \cdot \mathrm{ml}^{-1}$ in isopropanol and then vacuum filtrated through a PTFE filter membrane (Millipore, $100 \mathrm{~nm}$ pore size). After full evaporation of the solvent, the electrodes were cut from the AgNW/PTFE membrane into the required dimensions. The electrodes were then pasted on top of the rubber specimen (32 $\mathrm{mm} \mathrm{x}$ $9 \mathrm{~mm} \times 0.7 \mathrm{~mm}$ ) and a uniform pressure was applied for approximately 10 seconds. Finally the PTFE membrane was peeled off leaving the AgNW electrodes firmly attached to the specimen surface. The surface area density of the AgNW electrode was around $490 \mathrm{mg} \cdot \mathrm{m}^{-2}$ with a surface conductance of around $20 \mathrm{ohm} \cdot$ square $^{-1}$. As shown in Figure 1, the ends of AgNW electrodes were then glued to very thin, varnished wires (around $30 \mathrm{~mm}$ long) by conductive epoxy for further connection to a Keysight E4980 LCR meter. (B). Simultaneous test: As illustrated in Figure 1, 
samples were firstly glued to metal fixtures which were then clamped into a TA Instruments Q800 Dynamic Mechanical Analyzer (DMA), in order to avoid any prestraining of the sample. Electrically insulating tape was attached to the metal fixture to prevent electrical contact between the sample and the DMA. A uniaxial, multihysteresis strain profile was applied to the sample by the DMA while collecting impedance data at electrical frequencies of $1,10,100,1000 \mathrm{kHz}$ using the LCR meter. The multi-hysteresis profile consisted of a linear ramp to $0.1 \%$ strain followed by a linear ramp down to $0 \%$ strain. This cycle was repeated 3 times before loading to higher strains and cycling up to a maximum of $50 \%$ strain. The strain cycle frequency was $0.05 \mathrm{~Hz}$. Only data measured at the maximum applied strain for each first cycle is presented except where otherwise stated. All the data were averaged from at least three repeats except for those special illustrated.

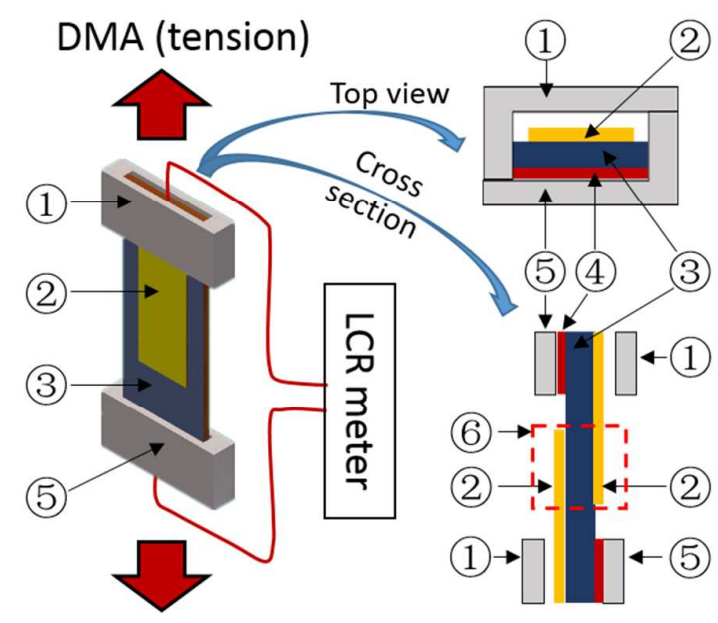

Figure 1. Setup of the simultaneous dielectric-mechanical test (SDMS). A top view and a cross sectional view of the specimen gives details of its assembly. Numbers refer to: 1. Top fixture; 2. AgNW electrode; 3. Specimen under test; 4. Glue; 5. Bottom fixture (specimens are glued to this type of fixture). 6. Material overlapped by AgNW electrodes. 


\section{RESULT AND DISCUSSION}

3.1. Carbon Black Networks in NR. Table 1 summarizes the sample codes and corresponding volume fractions used in this study. The N330 carbon black (CB) had a nitrogen-specific surface area of $77 \mathrm{~m}^{2} \cdot \mathrm{g}^{-1}$. In rubber compounds, CB typically forms different levels of network structure depending primarily on the CB volume fraction, $\phi$. The dc electrical conductivity, $\sigma_{d c}$, of filled compounds under static mechanical conditions has been widely used to investigate such CB structures. ${ }^{42-44}$ Figure 2 shows the $\sigma_{d c}-\phi$ trend for the samples used in this study. For low values of $\phi$, the material is non-conductive and filler aggregates form only non-percolated, discontinuous filler networks (sub-clusters) during the curing process. For example, the inset SEM picture in Figure 2 shows a cryogenic fracture surface image of NR/0.071 as an illustration of the filler dispersion in the rubber compound where only isolated $\mathrm{CB}$ aggregates and sub-clusters can be observed. However, when $\phi$ approaches a critical value, known as the apparent percolation threshold, $\phi_{c}^{d c}$, the dc conductivity is found to increase by several orders of magnitude (its absolute value is still low). This corresponds to the formation of a percolating network of filler particles throughout the material which establishes conductive pathways. Only when $\phi>\phi_{c}^{d c}$, does the dc conductivity become large (the material is essentially conductive) and it continues to increase somewhat with increasing $\phi$ due to further jamming of the filler network. 


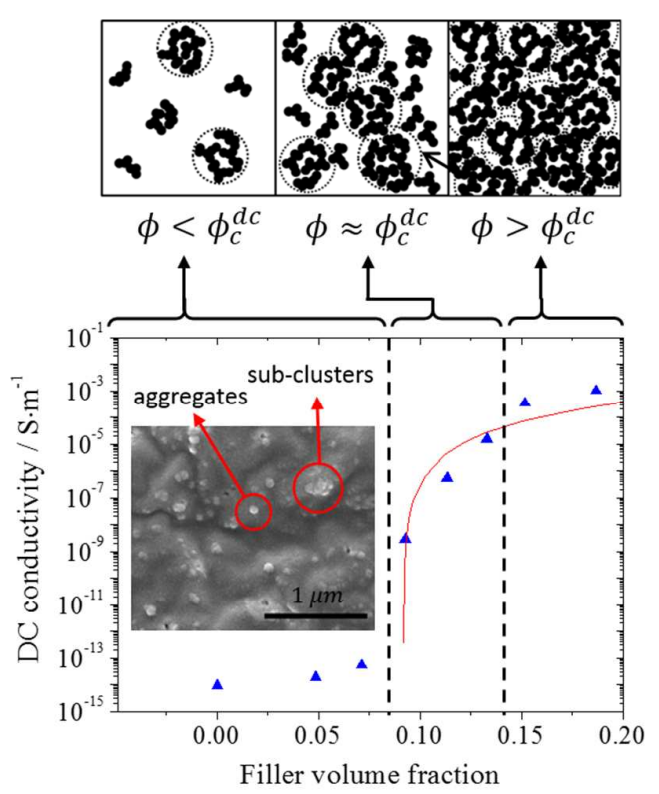

Figure 2. DC percolation trend for carbon black N330 filled NR. The inset graph is a SEM cryo-fracture surface image of the state of aggregate distribution throughout the NR matrix for sample NR/0.071.

The non-percolated filler networks exhibit a relatively low dielectric loss in a typical frequency range between $1 \mathrm{kHz}$ and $1 \mathrm{MHz}$ used in this study. Such low $D$ values are very important for obtaining accurate values of $\kappa^{*}$ via the impedance method. This is because the $\kappa^{*}$ derived from impedance methods always have multisolutions which depend on the magnitude of $D$. The difference between those solutions, can be ignored when $D$ is small $(D<0.1)$. But huge differences in $\kappa^{*}$ exist when $D$ is large (for example: difference $>100 \%$ in $\kappa^{*}$ when $D>1$ ). For such cases, one can only focus on the general trend of $\kappa^{*}$ as a function of, for example, temperature, frequency or strain rather than the absolute value. Therefore, for the dielectric measurement in this paper, we will only focus on the samples with relatively low dc conductivity and low dielectric loss $(\phi<0.133)$. 


\subsection{Effects of Filler Dispersion Quality on Dielectric Properties of Unstrained}

NR/CB. The dielectric behavior of filled NR is highly dependent on the state of filler dispersion. ${ }^{43}$ Figure 3(a) shows $\kappa^{\prime}$ and $D$ as a function of electrical frequency $(f)$ for $\mathrm{NR} / 0.13$ with varying levels of carbon black dispersion. In this case the dispersion state was controlled by increasing the mixing time on a 2-roll mill (50, 100 and 300 passes through the rollers set at a gap distance of around $0.2 \mathrm{~mm}$ ). As the dispersion state improves with the increased mixing energy, higher levels of interfacial MWSdipoles and CHARGE-dipoles is achieved corresponding to increased polymer-filler interaction area. Consequently both $\kappa^{\prime}$ and $D$ values increase with increasing dispersion quality. However, these effects are reduced with increasing frequency. As shown in Figure 3(a), both the $\kappa^{\prime}$ and $D$ values of different samples coalesce at higher frequencies. In the filled compounds the carbon black particles are highly conductive and the NR matrix surrounding the particles is insulating. This structure is somewhat analogous to ceramic materials with colossal $\kappa^{\prime}$, where grains are much more conductive than grain boundaries. Lunkenheimer et al. ${ }^{45}$ concluded that such high $\kappa^{\prime}$ is mainly caused by a strong MWS effect at the interface rather than the intrinsic dielectric properties of the component materials. Geberth and Klüppel ${ }^{32}$ observed similar behavior in the $\kappa^{\prime}-f$ spectrum of various carbon black reinforced-rubber compounds. They described this process as a Debye type relaxation, controlled by the inter-aggregate distance. However, it remains an open question as to whether this dielectric relaxation of $\mathrm{CB} /$ rubber composites originates from the intrinsic properties of the constituent materials or is caused by MWS polarization effects. 

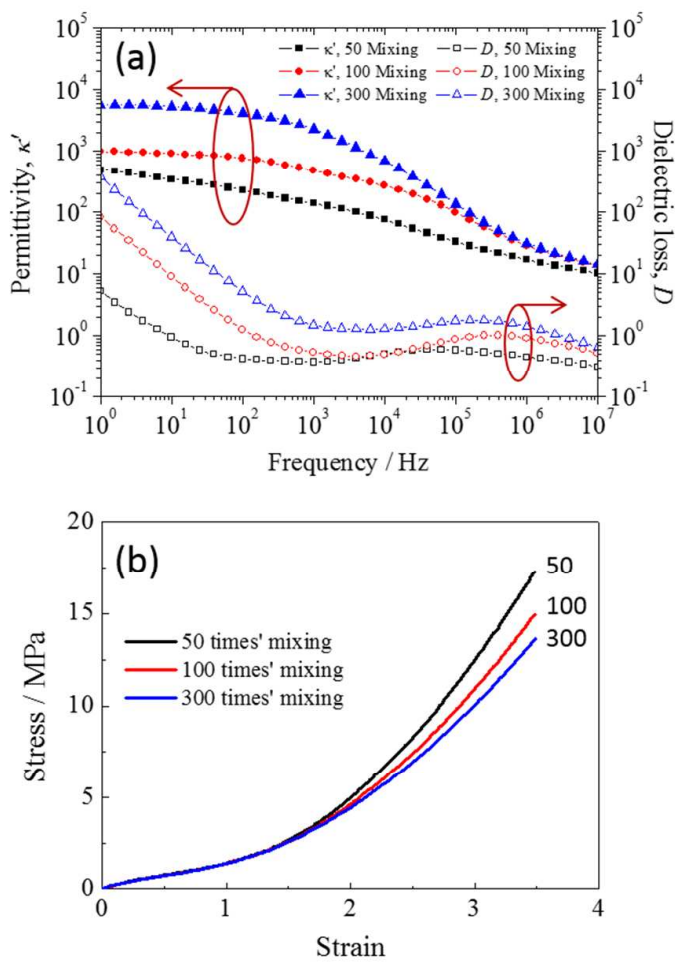

Figure 3. (a) Influence of mixing time on the measured dielectric properties. Samples are NR/0.13 mixed 50 times/100 times/300 times by two roll mill, named as $50 \mathrm{mix}$, 100 mix and 300 mix respectively. (b) Uniaxial stress-strain behavior of the same samples.

Figure 3(b) shows the tensile stress-strain behavior of these materials. In the strain range below $150 \%$, the mechanical behavior of the materials with varying filler dispersion states are identical. However, at strains above $150 \%$ significant differences are observed - with the better dispersed material showing a reduced incremental modulus. Probable explanations for this may be related to the shear modification of polymer chain length during mixing which may affect the crosslinked rubber network structure and therefore the ability of the material to strain crystallize. Additionally the improved dispersion of the filler aggregates may alter the magnitude 
of local strain amplification effects which may again affect the strain crystallization process. The data presented in Figure 3 demonstrates that the dielectric method is far more sensitive to the dispersed filler structures when compared with more traditional mechanical measurements. For all other samples discussed in this paper, the mixing process were controlled on a 2-roll mill at 100 roller passes.

3.3. Simultaneous Dielectric-Mechanical Spectroscopy (SDMS). The sensitivity limit of the SDMS testing system (Figure 1) can be seen in Figure 4(a) which shows the results of unfilled NR tested using the multi-hysteresis loading profile illustrated at an electrical frequency of $1 \mathrm{kHz}$. The LCR meter can resolve changes in capacitance above approximately $0.005 \mathrm{pF}$ at $1 \mathrm{kHz}$. This high level of resolution covers all the testing range except the data collected below $0.5 \%$ strain at $1 \mathrm{kHz}$ for $\mathrm{NR} / 0$ and NR/0.049. It is however, important to note that the absolute magnitude of $\kappa^{\prime}$ from the SDMS technique can be affected by inadvertent pre-straining of the sample, which in practice can never be fully avoided. This is directly analogous to strain history effects often observed in the small strain dynamic and static mechanical properties of filled compounds. ${ }^{44}$ 

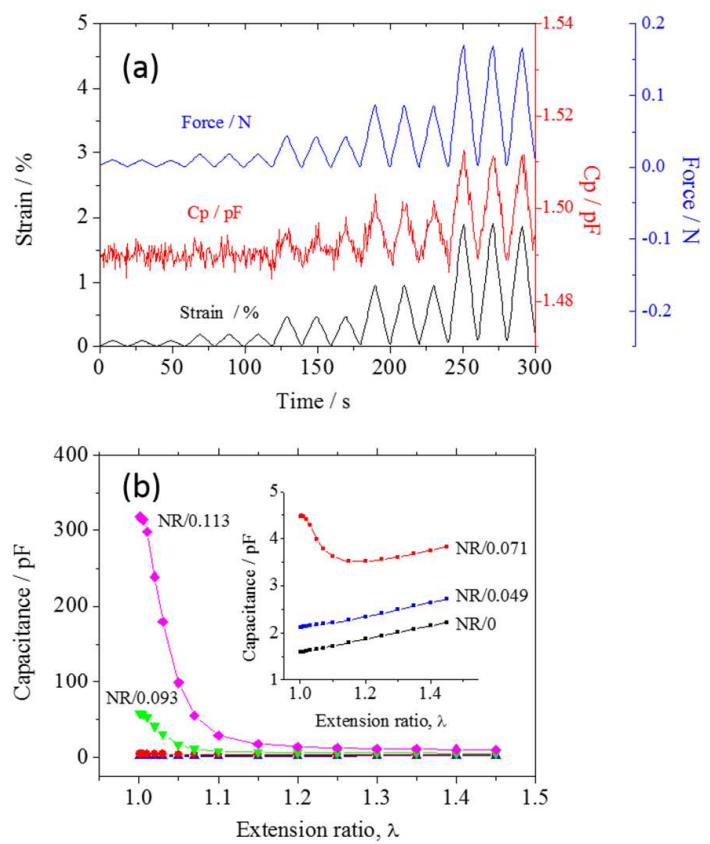

Figure 4. (a) Sensitivity limits of the SDMS method. The data is based on a single SDMS test of unfilled NR. All the dielectric data was measured at $1 \mathrm{kHz}$ electrical frequency. (b) Measured capacitance under strain for different samples. The inset graph shows a magnification of data for NR/0, NR/0.049 and NR/0.071.

In the SMDS test, as shown in Figure 1 (labeled as No.6), the capacitance of a sample having an overlapped sandwich structure under tensile deformation is proportional to the extension ratio, $\lambda$, and $\kappa^{\prime}$ of the material: ${ }^{46}$

$$
C_{p}=\kappa_{v a c} \kappa^{\prime} \lambda l_{0} w_{0} / d_{0}+C_{c o r r}
$$

where $C_{p}$ is the measured capacitance based on an RC parallel model. $l_{0}, w_{0}$ and $h_{0}$ refer to the unstrained length, width and thickness of the specimen respectively. $\kappa_{v a c}$ is the permittivity of a vacuum and $C_{c o r r}$ is the correction for capacitance which is caused by the non-uniform electrical field around the electrode edge as well as stray capacitance from the connection. This term can be treated as a constant throughout the test. From Equation $1, \kappa^{\prime}$ of the strained material can be calculated. For unfilled 
polymers such as NR or PDMS, $\kappa^{\prime}$ is independent of the applied strain up to at least $100 \%{ }^{46}$ In such cases, $C_{p}$ should be linearly proportional to the extension ratio, $\lambda$. Figure 4(b) (inset) confirms a linear $C_{p}$ versus $\lambda$ relationship measured for unfilled NR using the SDMS technique. Figure 4 (b) also shows the breakdown of this linear relationship as the filler volume fraction is increased. It demonstrates that $\kappa^{\prime}$ of these filled materials is not constant under the application of strain.

3.4. SDMS of Unfilled NR. CV60 grade NR typically consists of around 95\% cispolyisoprene (PI) polymer and a few percent of other components such as proteins, fatty acids. ${ }^{47}$ In dielectric terms PI has possesses both A-type and B-type dipoles. They are responsible for the 'slow' chain relaxation $(\beta)$ and 'fast' segmental relaxation ( $\alpha$ ) respectively. ${ }^{48}$ Figure 5 shows $\kappa^{\prime}$ and $D$, measured as a function of strain for $\mathrm{NR} / 0$. In Figure 5 the large red stars refer to data obtained at $1 \mathrm{kHz}$ by a standard dielectric method (Section 2.4 A). As can be seen, the data measured at small $(0.1 \%)$ strain by the SDMS technique tends towards that measured at $0 \%$ strain by the standard impedance method. This indicates that the data obtained by the SDMS method at small strains are reliable. NR/0 always shows a strain independent $\kappa^{\prime}$ for all the testing frequencies in each repeat test. The versy small discrepancy between the values is within the error associated with either the calibration of the equipment or the accuracy of the measurements of the sample geometry.

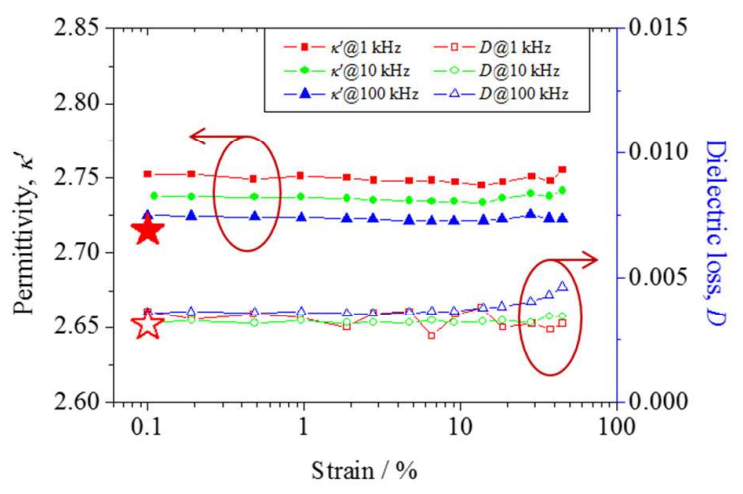


Figure 5. The dielectric behavior of unfilled NR as a function of strain. The filled red star is $\kappa^{\prime}$ measured by Novocontrol Concept 40 Dielectric Spectrometer at $1 \mathrm{kHz}$ and the hollow star is the corresponding dielectric loss data, $D$.

Due to the multi-component composition of NR, it possesses a broad distribution of dipole timescales. The dielectric properties of unfilled NR are highly dependent on electrical frequency. In the frequency domain $\kappa^{\prime}$ decreases with increasing frequency (Figure 5). Conversely, the dielectric properties of unfilled NR are essentially independent of applied strain at a fixed frequency (Figure 5). Lee et al. ${ }^{38}$ reported that the dielectric segmental relaxation was independent of strain for cured synthetic polyisoprene. But Hernández et al.$^{34}$ found an increase of the dielectric strength with strain for cured NR in conflict with Lee's results. They explained this increase was a consequence of increased dipole moments of the polymer under strain. For our materials, although the rubber chains are aligned and confined in the direction of tension, there is very little change in the overall dielectric response. This may because the strains in our test are too small to develop any additional dielectric response. Moreover, Lee and Hernández's results were derived from the imaginary part of permittivity and a detailed HN fitting. The $\kappa^{\prime}$ and $D$ collected using our SDMS technique is probably more accurate. This suggest that the BULK-diploes are independent of strain over the range tested here.

3.5. SDMS of Carbon Black Reinforced NR. The dielectric behavior of NR becomes significantly more complex when carbon black particles are introduced. As reviewed in the introduction section, at least five types of dipole may contribute to the observed permittivity under any given electrical frequency or deformation condition. As these different dipoles operate over differing length and time scales, it is possible 
to use the electrical frequency and/or the mechanical deformation as 'dipole filters' in order to discriminate between the different dipole relaxation timescales as a function of filler volume fraction.

Figure 6(a) details the $\kappa^{\prime}$ of natural rubber as a function of carbon black volume fraction under various electrical frequency and deformation conditions. The data collected for the samples at $1 \mathrm{kHz}$ under $0.1 \%, 10 \%$ and $45 \%$ strain are indicated by black squares, green inverse triangles and red circles respectively. Finally the $\kappa^{\prime}$ measured under the no-strain condition at $40 \mathrm{GHz}$ are indicated by the blue triangles. It is clear from Figure 6(a) that the measured $\kappa^{\prime}$ of carbon black-reinforced NR depends upon the filler volume fraction, the electrical frequency and the applied strain. Figure 6(a) can be broken down into linear combinations of certain types of dipoles:
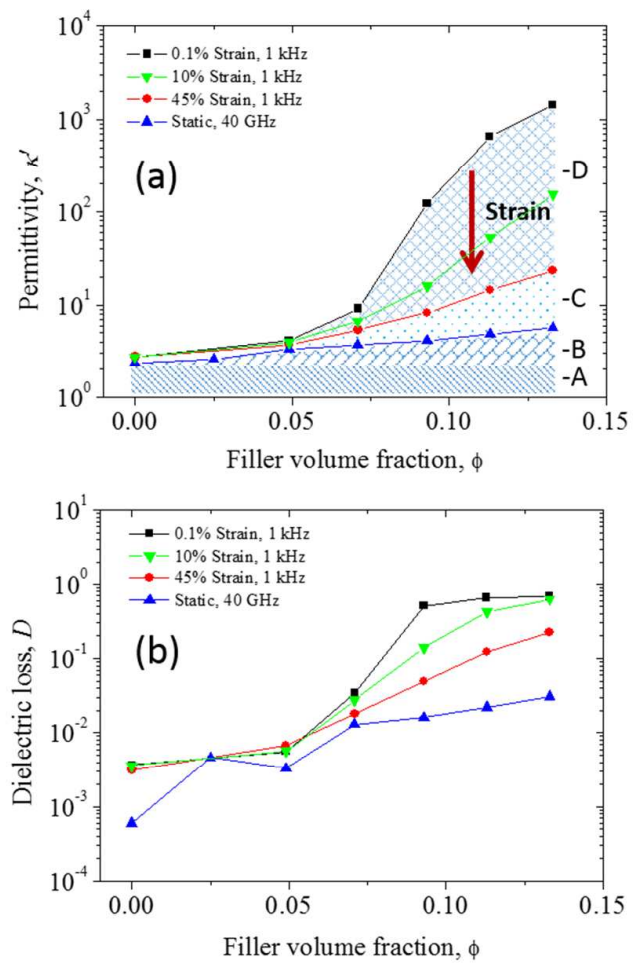
Figure 6. (a) The relationship between the real part of permittivity $\kappa^{\prime}$ and filler volume fraction $\phi$ under different frequencies and strain conditions. (b) Dielectric loss against $\phi$ under different conditions.

Zone A: This region refers to the bulk polarization of the NR matrix at an electrical frequency of $40 \mathrm{GHz}$. In fact, all samples (filled and unfilled) show very limited frequency dependent dielectric behavior when the electrical frequency is above 7 GHz. Further to this we also find that at such high frequencies there is no systematic dependence of $\kappa^{*}$ on the filler surface area which contrasts with other literature work performed at much lower electrical frequencies ${ }^{49}$ (See Figure S1 in the supporting materials). This means that dielectric contributions from MWS-dipoles and CHARGE-dipoles, which are highly dependent on the quantity of polymer-filler interfacial area, are filtered out at higher frequencies and can be ignored in the high frequency range.

Zone B: the $\kappa^{\prime}$ collected at $40 \mathrm{GHz}$ under no-strain conditions displays an increasing logarithmic relationship between $\kappa^{\prime}$ and the filler volume fraction. Zone B can therefore only consist of contributions from GLASSY-dipoles and FILLERdipoles since the electrical frequency is too high to observe any contribution from MWS-dipoles and CHARGE-dipoles. However, in previous work on similar materials, no evidence for the existence of glassy/immobilized polymer domains was found. ${ }^{24}$ This could be due to the volumes of glassy/immobilized material being too small for observed by current testing technologies. In this case, we can treat the GLASSY-dipoles as being an insignificant contribution to the total dielectric behavior. 
Zones C and D: for filled materials the contributions from interfacial MWS diploes and CHARGE dipoles are substantial. For data collected at low frequency $(1 \mathrm{kHz})$ with no applied strain, the contributions from the diploes are at a maximum (the summation of Zone C and Zone D). However, we can see that when the mechanical strain is increased, the contributions from these dipoles $(1 \mathrm{kHz})$ decrease dramatically (indicated by the transition from Zone $\mathrm{D}$ to Zone $\mathrm{C}$ in Figure 6 (a)). Sow el al. ${ }^{27}$ demonstrated that mechanical strain can change the magnitude or even the direction of charge transfer between surfaces. Additionally, the MWS polarization is highly dependent on the morphology of the filler network ${ }^{15}$ which is also modified by the application of strain. ${ }^{42}$ In this sense the application of mechanical strain is analogous to an increase in electrical frequency.

Due to limitations with the testing set up, the maximum strain which could be applied was around $50 \%$. It is assumed that Zone $\mathrm{C}$ would systematically reduce in magnitude if even larger strains were applied. Therefore a depleted Zone C refers to strain-independent dipoles such as FILLER-dipoles and some of the CHARGEdipoles and MWS-dipoles. Figure 6(b) shows the dielectric loss for all the data presented in Figure 6(a). The maximum loss is around 0.68 which still allows us to discuss the relationship between $\kappa^{\prime}$ and strain with acceptable accuracy.

Figure 7(a) and Figure 7(b) show the whole SDMS spectra for the NR/0.071 sample. Thus the strain $(e: 0.1 \%-45 \%)$ and frequency $(f: 1 \mathrm{kHz}-1 \mathrm{MHz})$ dependent dielectric behavior of the material is constructed. Figure 7(c) and Figure 7(d) present projections of the data from Figure 7(a) and Figure 7(b) on different planes. In the $\kappa^{\prime}-e$ plane in Figure $7(\mathrm{c})$, the total strain dependence of $\kappa^{\prime}$ (that is $\left.\kappa_{e \rightarrow 45 \%}^{\prime}-\kappa_{e \rightarrow 0.1 \%}^{\prime}\right)$ is significantly larger at lower electrical frequencies. At the same time the total strain dependence of $D$ (that is $D_{e \rightarrow 45 \%}-D_{e \rightarrow 0.1 \%}$ ) becomes more 
significant at higher frequencies as shown in the $D-e$ plane in Figure 7(d). This behavior is due to the different timescales of the various dipoles and, as stated previously, there is a certain analogy between the role of strain and electrical frequency in suppressing dipolar contributions. Similarly, in the $f-e$ plane in Figure 7(c), the total frequency dependence of $\kappa^{\prime}$ (that is $\kappa_{f \rightarrow 1 \mathrm{kHz}}^{\prime}-\kappa_{f \rightarrow 1 \mathrm{MHz}}^{\prime}$ ) becomes much smaller at higher strain. In the $f-e$ plane in Figure $7(\mathrm{~d})$ the total strain frequency dependence of $D$ (that is $D_{e \rightarrow 1 \mathrm{k}}-D_{e \rightarrow 1 \mathrm{M}}$ ) is less significant at higher strains. This may be because most of the frequency-sensitive dipoles have already been filtered out by the application of strain. Figure 7 therefore shows that it is possible to 'filter' the contributions of dipoles to the strain-dependent dielectric properties of the material by simply tuning the frequency of applied electrical field. Unlike the limited range of strain rate accessible to DMA tests, electrical frequency in SDMS technology can be varied by orders of magnitude. This enables a very broad frequency filter for dipolar activity during the straining process.
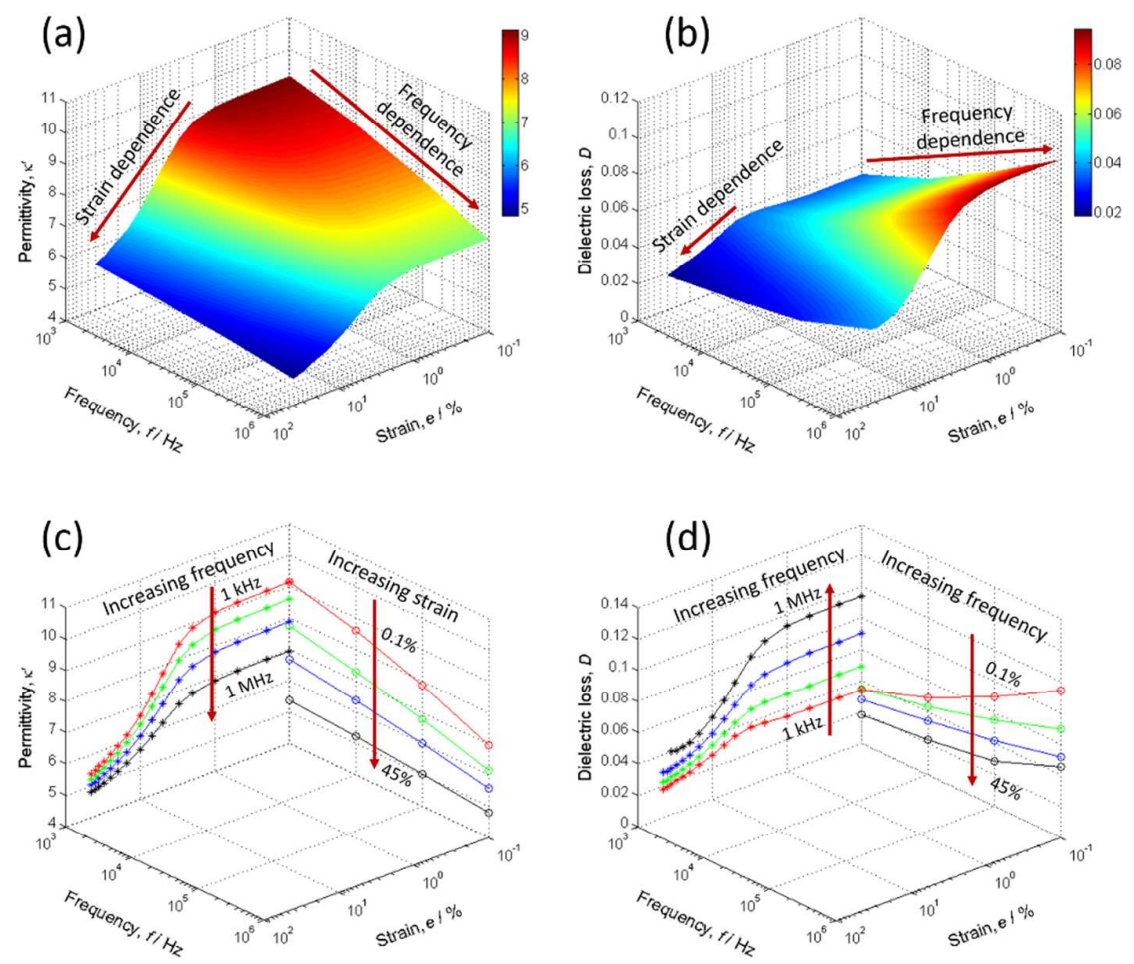
Figure 7. (a) \& (b) Strain $e$ and electrical frequency $f$ dependent behavior of $\kappa^{\prime}$ and $D$ of NR/0.071. (c) \& (d) Projections of data from (a) and (b) respectively with selected data. Solid lines connect the data measured at the same strain (frequency- $\kappa^{\prime}$ plane) or frequency (strain- $\kappa^{\prime}$ plane) as guides for the eye.

3.6. Dielectric vs Mechanical Spectroscopy. Figure 8(a) shows the dielectric and the mechanical behavior of NR/0.071 subjected to the multi-hysteresis strain profile as described in Section 2.5. The dielectric data measured at an electrical frequency of $1 \mathrm{kHz}$ is presented. Figure 8(b) and (c) are sub-data from Figure 8(a) of the cyclical softening sections of the multi-hysteresis strain sweep showing the three cycles undertaken between zero strain and a peak strain of 5\%. The dielectric loss of $\mathrm{NR} / 0.071$ is smaller than 0.1 for all the strains which confirms the physical meaning of the derived $\kappa^{\prime}$.
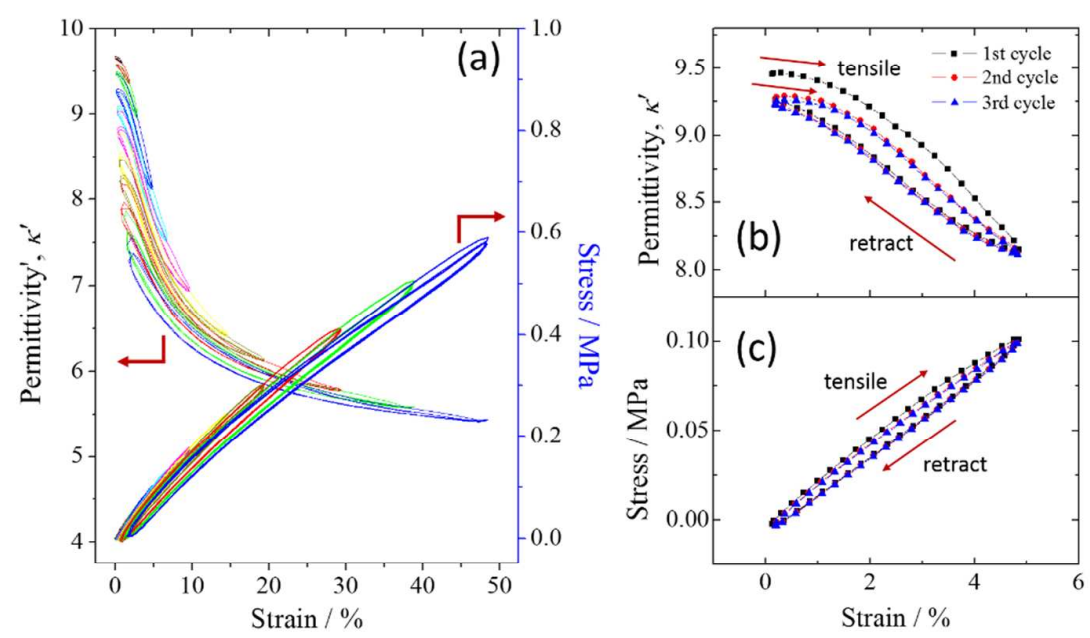

Figure 8. $\kappa^{\prime}$ and stress vs strain for NR/0.071. (a) Multi-hysteresis data from 0 to 50\% strain. (b) \& (c) Sub-data taken from the multi-hysteresis sweeps showing the 0- 
$5 \%$ cycle data more clearly. All SDMS data was measured at an electrical frequency of $1 \mathrm{kHz}$. All data collected during a test is presented.

As discussed in Section 3.5, $\kappa^{\prime}$ decreases with an increase in strain amplitude. The primary reason for this is the strain dependence of MWS-dipoles and CHARGEdipoles. However there are several other possible contributing and competing factors. For example, the filler aggregates can re-orientate with the direction of strain. ${ }^{42}$ Recent work by Wang et al. ${ }^{50}$ has shown that such re-orientation effects can be correlated with a decrease in $\kappa^{\prime}$. Other factors may even compete to increase the measured $\kappa^{\prime}$ under applied strain. For example, the strain-induced rupturing of the filler cluster-cluster agglomerates actually increases the filler-rubber interface area (and therefore the extent of the interfacial polarization) with increasing strain. Furthermore, there is a strain dependence on the level of occluded rubber in the filled materials. Occluded rubber is defined as the rubber polymer trapped between agglomerated filler clusters and thereby mechanically 'shielded' from the globally applied strain field and electromagnetically 'shielded' from the global alternating electrical field. ${ }^{51}$ As the agglomerated filler clusters break down under strain, this occluded rubber is progressively exposed to the global mechanical and electrical fields. Therefore, the strain induced dielectric behavior is a result of competition of all those possible mechanisms mentioned above. Examining the phenomenology of the dielectric response of the material under strain we can make the following observations:

- The magnitude of the strain-induced decrease in $\kappa^{\prime}$ depends on the largest strain applied to the sample. 
- When strain is removed, $\kappa^{\prime}$ can only partially recover its initial small strain magnitude and does not fully recover its original magnitude (at least within the experimental timescale).

- The cyclic strain dependence of $\kappa^{\prime}$ becomes increasingly reversible after the initial cycle at a specific value of maximum strain (Figure 8(b)). A dielectric hysteresis is apparent between loading and unloading cycles.

This behavior is analogous to the simultaneously measured mechanical behavior shown in Figure 8(a) and Figure 8(c) which exhibits the typical 'Mullins' effect and cyclic stress softening responses to multi-hysteresis testing. This similarity arises from the structural dynamics of the filler network and the filler-polymer interaction. However, the dielectric behavior is dominated by dielectric phenomena at the polymer-filler interface rather than the bulk polymer. The full potential of the interfacial information extracted from such new experiments is currently under exploration.

Figure 9 shows both mechanical and dielectric data measured simultaneously for the various volume fractions of carbon black. Figure 9(a) shows the classical 'Payne' effect for these materials with the characteristic strain-dependence of the tensile modulus becoming more apparent with increasing carbon black volume fraction. Similarly, Figure 9 (b) and Figure 9 (c) show a corresponding systematic straindependent decrease in both $\kappa^{\prime}$ and $D$. Clearly this can be seen as a dielectric analogy of the mechanical 'Payne' effect. Note that the strain dependence of the dielectric properties span several order of magnitude from small to high strains. This again indicates that the SDMS method is significantly more sensitive to microstructural dynamics in filled rubber than traditional mechanical testing. In Figure 9(c), although the dielectric loss data of these samples are increased by carbon black, it is still very 
1

2

3

4

5

10

useful to exploit this strain sensitivity of both $\kappa^{\prime}$ and $D$ to study the morphology of filler structures or to build strain sensors.

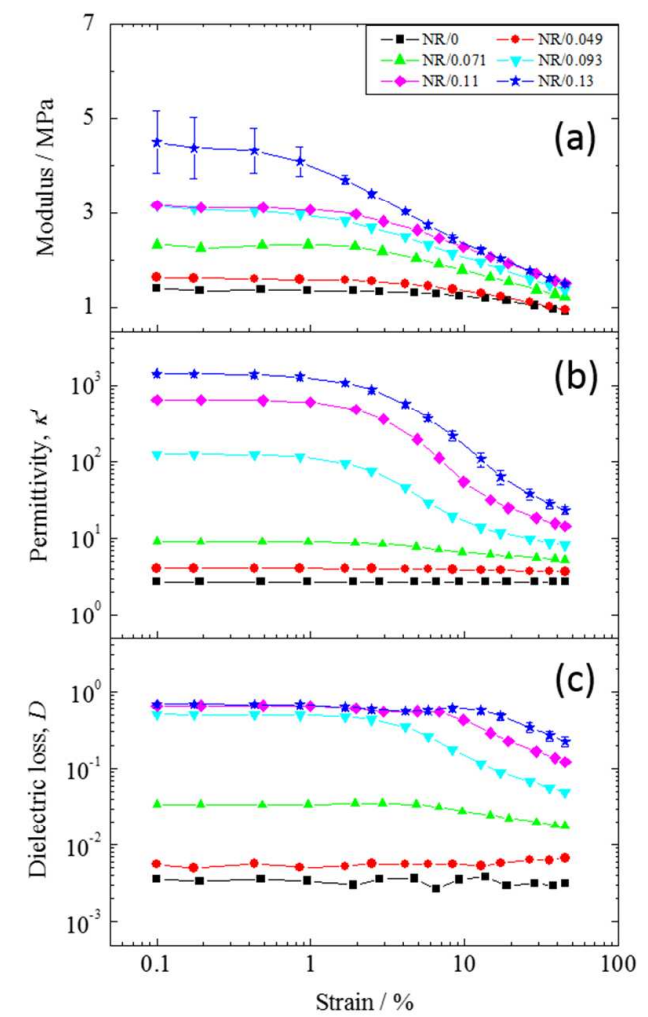

Figure 9. (a) Static modulus (stress/strain) vs strain collected simultaneously with the dielectric data. (b) \& (c) Strain-dependence of $\kappa^{\prime}$ and $D$ of all samples (measured at an electrical frequency of $1 \mathrm{kHz}$ ).

\section{CONCLUSION}

This paper presents a method to simultaneously characterize the dielectric and dynamic mechanical properties of rubber composites. Firstly it clearly demonstrates that, not only the mechanical modulus but also the permittivity display a nonlinear dependence on mechanical strain. Therefore the permittivity of nanocomposites (even with a very low filler concentration) is not a fixed "constant" (although it is also 
known as dielectric constant) but varies dramatically under even few percent applied strain. The underlying reason for this is the strain dependence of MWS-dipoles and CHARGE-dipoles at the interface. Secondly, dipoles around the interface region can be suppressed or 'filtered' by applying mechanical strain. Additionally, at each strain level the contributing dipoles can be further 'filtered' by modulating the electrical frequency. This technique provides an effective way to study the electrical structure of the interface region. Finally, in comparison with traditional mechanical testing, the dielectric spectrum is far more sensitive dependence on the structural dynamics of the compounds. This may be useful as a powerful tool in the tire and rubber industries for characterizing, understanding and monitoring of rubber products.

\section{ASSOCIATED CONTENT}

Supporting Information. This part includes extra dielectric data measured at $\mathrm{GHz}$ range. This material is available free of charge via the Internet at http://pubs.acs.org.

\section{AUTHOR INFORMATION}

\section{Corresponding Author}

*E-mail: j.busfield@qmul.ac.uk (J.J.C.B)

\section{Author Contributions}

The manuscript was written through contributions of all authors. All authors have given approval to the final version of the manuscript. 


\title{
ACKNOWLEDGMENTS
}

M.H. would like to thank the China Scholarship Council for doctoral funding and Cabot Corporation (Boston, USA) for kindly providing the materials. L.B.T thanks the Soft Matter Group at Queen Mary University of London for post-doctoral funding. The authors would like to thank Professor Jiaming Tang and Dr Gang Zhao from Xidian University in China for helping with the microwave characterization. The authors would also like to thank Professor Xiaoyong Wei from Xi'an Jiaotong University for discussions concerning the design of the test.

\begin{abstract}
ABBREVIATIONS
AgNW, silver nanowire; CB, carbon black; DMA, dynamic mechanical analyzer; NR, natural rubber; SDMS, simultaneous dielectric-mechanical spectroscopy.
\end{abstract}

\section{REFERENCES}

(1) Kraus, G. Rubber Chem. Technol. 1978, 51, 297-321.

(2) Wang, M.-J. Rubber Chem. Technol. 1998, 71, 520-589.

(3) Vilgis, T. A.; Heinrich, G.; Klüppel, M. Reinforcement of Polymer Nanocomposites: Theory, Experiments and Applications; Cambridge University Press: Cambridge, 2009.

(4) Dang, Z. M.; Yuan, J. K.; Yao, S. H.; Liao, R. J. Adv. Mater. 2013, 25, 63346365.

(5) Risse, S.; Kussmaul, B.; Krüger, H.; Kofod, G. Adv. Funct. Mater. 2012, 22, 3958-3962. 
(6) Chen, Y.-S.; Hsieh, G.-W.; Chen, S.-P.; Tseng, P.-Y.; Wang, C.-W. ACS Appl. Mater. Inter. 2015, 7, 45-50.

(7) Payne, A. R. J. Appl. Polym. Sci. 1962, 6, 57-63.

(8) Mullins, L. Rubber Chem. Technol. 1969, 42, 339-362.

(9) Heinrich, G.; Klüppel, M. Recent Advances in the Theory of Filler Networking in Elastomers. In Filled Elastomers Drug Delivery Systems; Springer: Berlin, 2002; pp 1-44.

(10) Maier, P.; Göritz, D. Kautsch. Gummi Kunstst. 1996, 49, 18-21.

(11) Merabia, S.; Sotta, P.; Long, D. R. Macromolecules 2008, 41, 8252-8266.

(12) Robertson, C. G.; Wang, X. Phys. Rev. Lett. 2005, 95, 075703.

(13) Wang, X.; Robertson, C. G. Phys. Rev. E 2005, 72, 031406.

(14) Van Beek, L. K. H. Dielectric Behaviour of Heterogeneous Systems. In Progress in Dielectrics; Heywood Books: London, 1967; Vol. 7, pp 69-114.

(15) Steeman, P.; Van Turnhout, J. Dielectric Properties of Inhomogeneous Media. In Broadband Dielectric Spectroscopy; Kremer, F.; Schönhals, A., Eds.; Springer: Berlin, 2003; pp 495-522.

(16) Lewis, T. J. IEEE Trans. Dielectr. Electri. Insul. 1994, 1, 812-825.

(17) Tanaka, T.; Kozako, M.; Fuse, N.; Ohki, Y. IEEE Trans. Dielectr. Electri. Insul. 2005, 12, 669-681.

(18) Stockmayer, W. H. Pure Appl. Chem. 1967, 15, 539-554. 
(19) Bansal, A.; Yang, H.; Li, C.; Cho, K.; Benicewicz, B. C.; Kumar, S. K.; Schadler, L. S. Nat. Mater. 2005, 4, 693-698.

(20) Berriot, J.; Montes, H.; Lequeux, F.; Long, D.; Sotta, P. Macromolecules 2002, 35, 9756-9762.

(21) Fragiadakis, D.; Bokobza, L.; Pissis, P. Polymer 2011, 52, 3175-3182.

(22) Mujtaba, A.; Keller, M.; Ilisch, S.; Radusch, H.-J.; Beiner, M.; ThurnAlbrecht, T.; Saalwächter, K. ACS Macro Lett. 2014, 3, 481-485.

(23) Robertson, C.; Roland, C. Rubber Chem. Technol. 2008, 81, 506-522.

(24) Huang, M.; Tunnicliffe, L. B.; Thomas, A. G.; Busfield, J. J. C. Eur. Polym. J. 2015, 67, 232-241.

(25) Ishii, H.; Sugiyama, K.; Ito, E.; Seki, K. Adv. Mater. 1999, 11, 605-625.

(26) Bard, A. J.; Faulkner, L. R. Double-Layer Structure and Adsorption. In Electrochemical Methods: Fundamentals and Applications, Second Edition; Wiley: New York, 2001.

(27) Sow, M.; Widenor, R.; Kumar, A.; Lee, S. W.; Lacks, D. J.; Sankaran, R. M. Angew. Chem. 2012, 124, 2749-2751.

(28) Maxwell, J. C. A Treatise on Electricity and Magnetism; Oxford University Press: London, 1873; Vol. 1.

(29) Wagner, K. W. Arch. Electrotech. 1914, 2, 371-387.

(30) Sillars, R. J. Inst. Elect. Eng. 1937, 12, 139-155.

(31) Pötschke, P.; Dudkin, S. M.; Alig, I. Polymer 2003, 44, 5023-5030. 
(32) Geberth, E.; Klüppel, M. Macromol. Mater. Eng. 2012, 297, 914-922.

(33) Dang, Z.-M.; Yao, S.-H.; Xu, H.-P. Appl. Phys. Lett. 2007, 90, 012907.

(34) Hernández, M.; López-Manchado, M. A.; Sanz, A.; Nogales, A.; Ezquerra, T. A. Macromolecules 2011, 44, 6574-6580.

(35) Raihan, R.; Adkins, J.-M.; Baker, J.; Rabbi, F.; Reifsnider, K. Compos. Sci. Technol. 2014, 105, 160-165.

(36) Brosseau, C.; Talbot, P. Meas. Sci. Technol. 2005, 16, 1823-1832.

(37) Wissler, M.; Mazza, E. Sens. Actuators, A: Phys. 2007, 138, 384-393.

(38) Lee, H.; Fragiadakis, D.; Martin, D.; Milne, A.; Milne, J.; Runt, J. Macromolecules 2010, 43, 3125-3127.

(39) Lee, P.; Lee, J.; Lee, H.; Yeo, J.; Hong, S.; Nam, K. H.; Lee, D.; Lee, S. S.; Ko, S. H. Adv. Mater. 2012, 24, 3326-3332.

(40) Hammock, M. L.; Chortos, A.; Tee, B. C. K.; Tok, J. B. H.; Bao, Z. Adv. Mater. 2013, 25, 5997-6038.

(41) Baker-Jarvis, J.; Vanzura, E. J.; Kissick, W. IEEE Trans. Microwave Theory Tech. 1990, 38, 1096-1103.

(42) Yamaguchi, K.; Busfield, J. J. C.; Thomas, A. G. J. Polym. Sci., Part B: Polym. Phys. 2003, 41, 2079-2089.

(43) Nan, C.-W.; Shen, Y.; Ma, J. Annu. Rev. Mater. Res. 2010, 40, 131-151.

(44) Tunnicliffe, L. B.; Kadlcak, J.; Morris, M. D.; Shi, Y.; Thomas, A. G.; Busfield, J. J. C. Macromol. Mater. Eng. 2014, 299, 1474-1483. 
(45) Lunkenheimer, P.; Bobnar, V.; Pronin, A.; Ritus, A.; Volkov, A.; Loidl, A. Phys. Rev. B 2002, 66, 052105.

(46) Cohen, D. J.; Mitra, D.; Peterson, K.; Maharbiz, M. M. Nano Lett. 2012, 12 , 1821-1825.

(47) Bateman, L. Chemistry and Physics of Rubber-Like Substances; Maclaren: London, 1963.

(48) Boese, D.; Kremer, F. Macromolecules 1990, 23, 829-835.

(49) Dang, Z.-M.; Yuan, J.-K.; Zha, J.-W.; Zhou, T.; Li, S.-T.; Hu, G.-H. Prog. Mater. Sci. 2012, 57, 660-723.

(50) Wang, Y.; Kim, S.; Li, G. P.; Sun, L. Z. Comp. Mater. Sci. 2015, 104, 69-75.

(51) Medalia, A. I. Rubber Chem. Technol. 1986, 59, 432-454. 


\section{for Table of Contents use only}

\section{Strain Dependent Dielectric Behavior of Carbon Black Reinforced Natural Rubber}

Menglong Huang, ${ }^{\dagger,+}$ Lewis B. Tunnicliffe, ${ }^{\dagger, t}$ Jian Zhuang, ${ }^{\S}$ Wei Ren ${ }^{\S}$ Haixue Yan, ${ }^{\dagger, \hbar}$ James J.C. Busfield ${ }^{*}+$, t
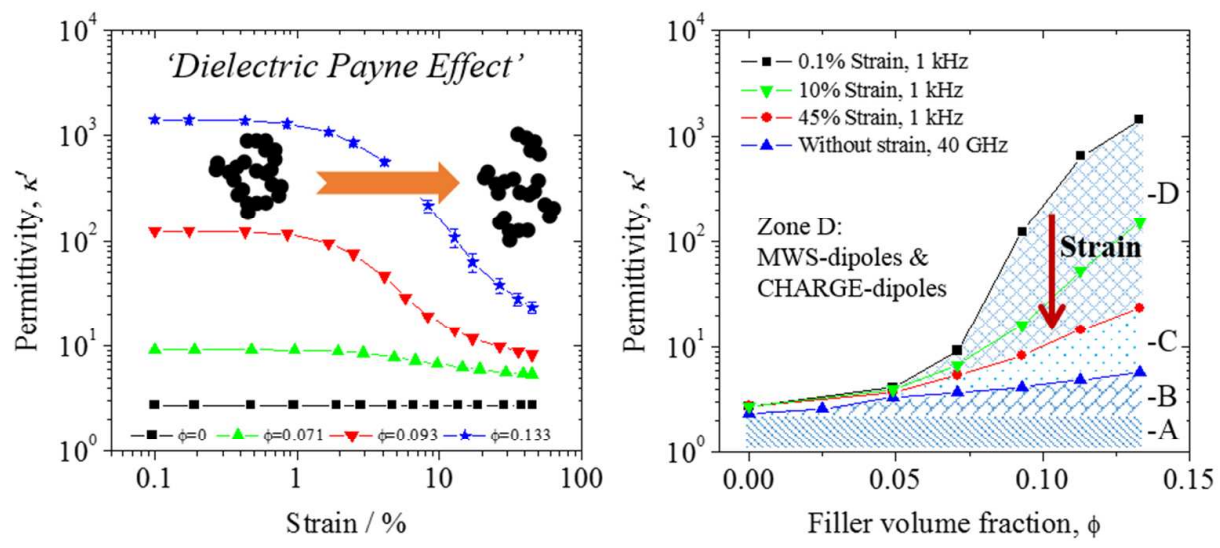

34

35

36

37

38

39

40

41

42

43

44

45

46

47

48

49

50

51

52

53

54

55

56

57

58

59 


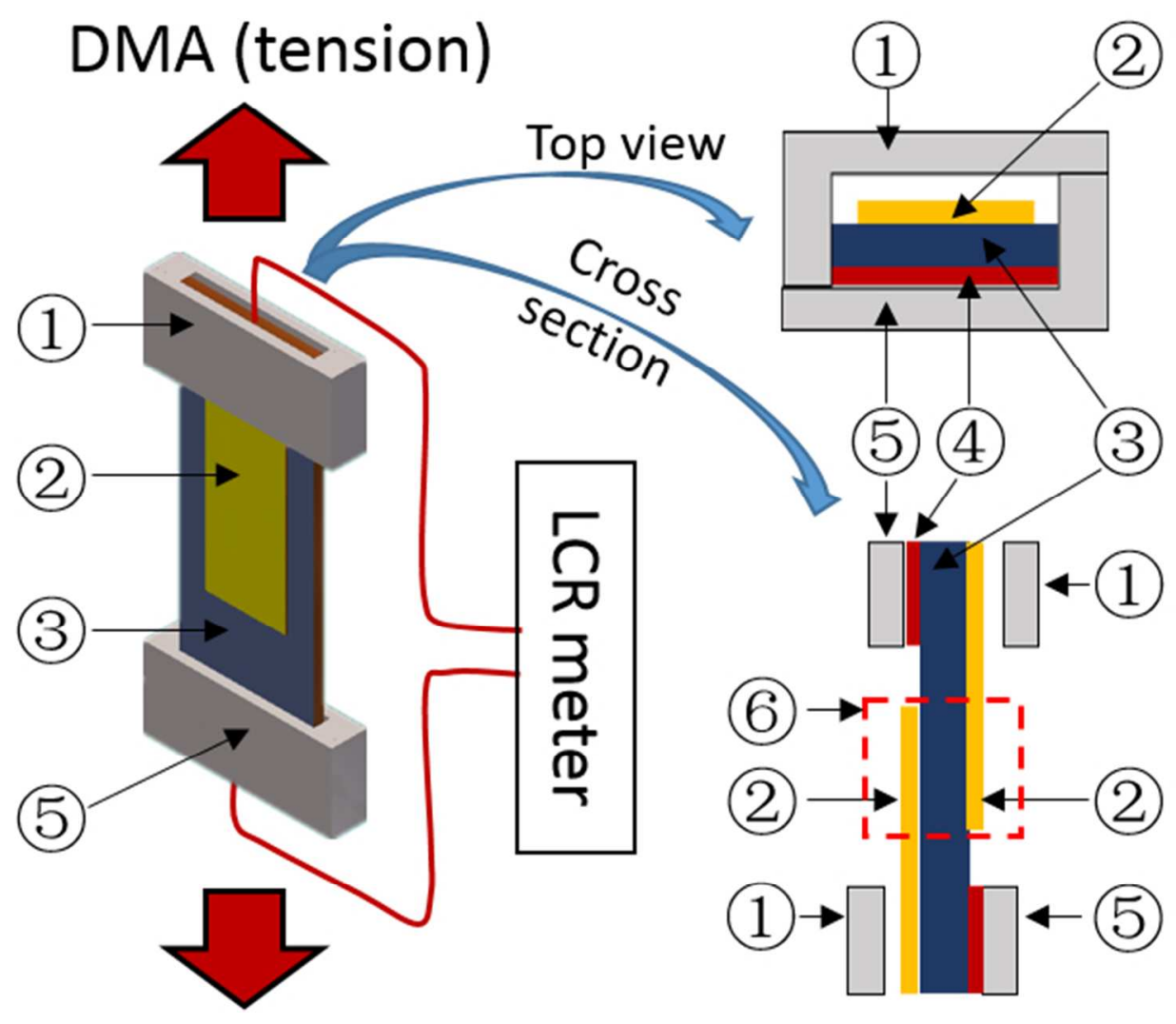

Figure 1. Setup of the simultaneous dielectric-mechanical test (SDMS). A top view and a cross sectional view of the specimen gives details of its assembly. Numbers refer to: 1 . Top fixture; 2 . AgNW electrode; 3. Specimen under test; 4. Glue; 5. Bottom fixture (specimens are glued to this type of fixture). 6 . Material overlapped by AgNW electrodes. $80 \times 66 \mathrm{~mm}(204 \times 204$ DPI $)$ 


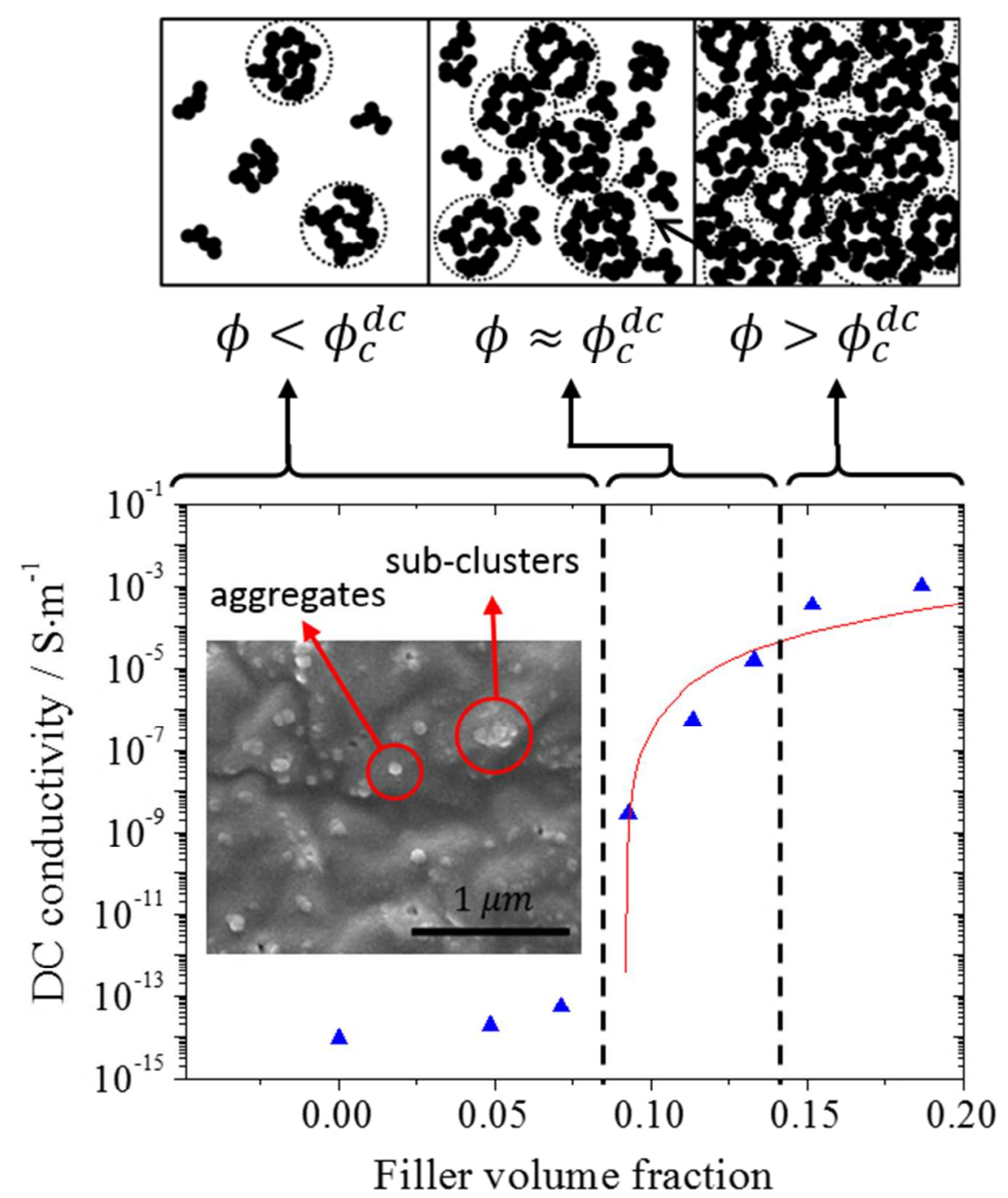

Figure 2. DC percolation trend for carbon black N330 filled NR. The inset graph is a SEM cryo-fracture surface image of the state of aggregate distribution throughout the NR matrix for sample NR/0.071. $80 \times 85 \mathrm{~mm}(224 \times 224$ DPI $)$ 

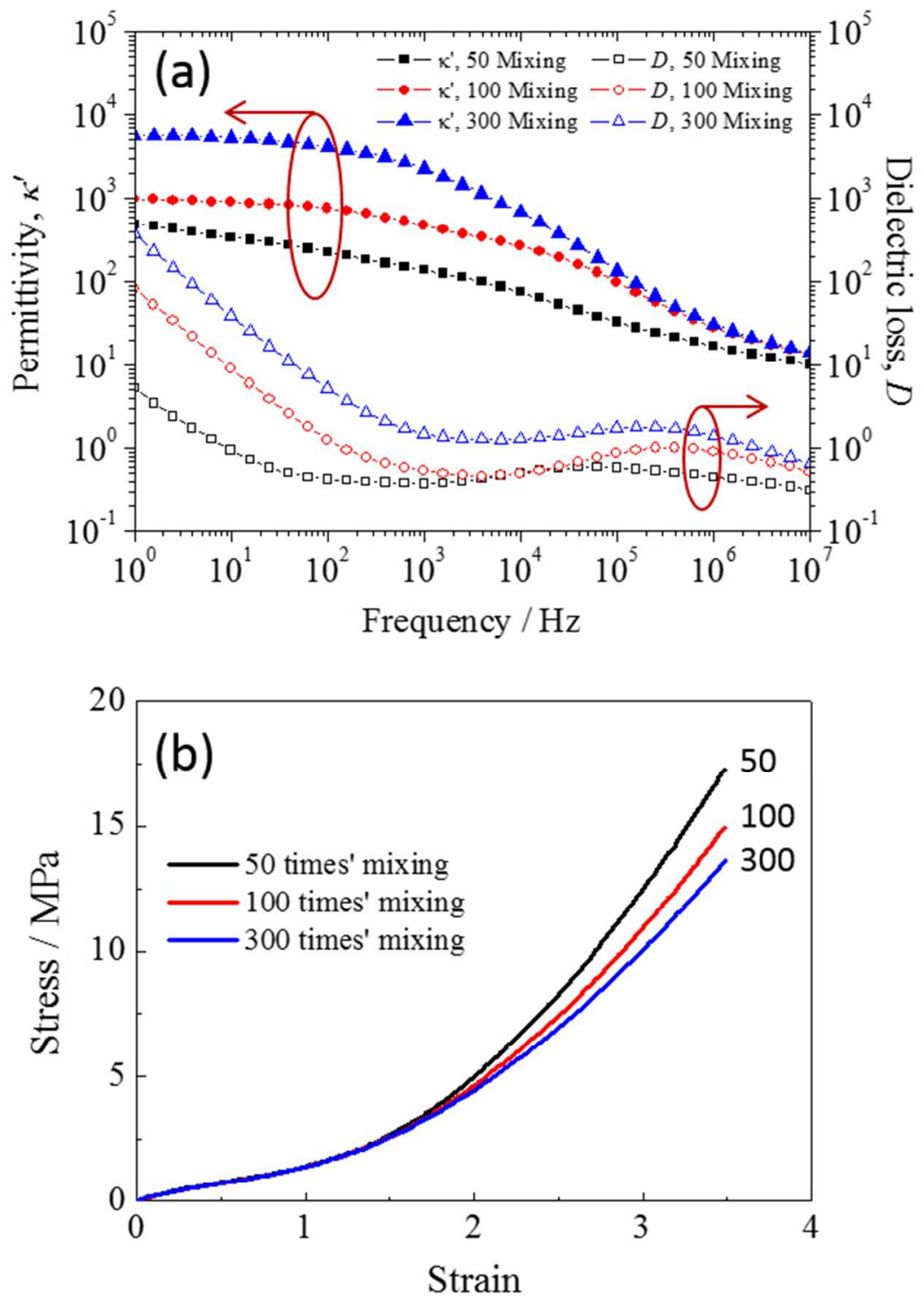

Figure 3. (a) Influence of mixing time on the measured dielectric properties. Samples are NR/0.13 mixed 50 times/100 times/300 times by two roll mill, named as $50 \mathrm{mix}, 100 \mathrm{mix}$ and $300 \mathrm{mix}$ respectively. (b) Uniaxial stress-strain behavior of the same samples. $80 \times 108 \mathrm{~mm}(218 \times 218 \mathrm{DPI})$ 

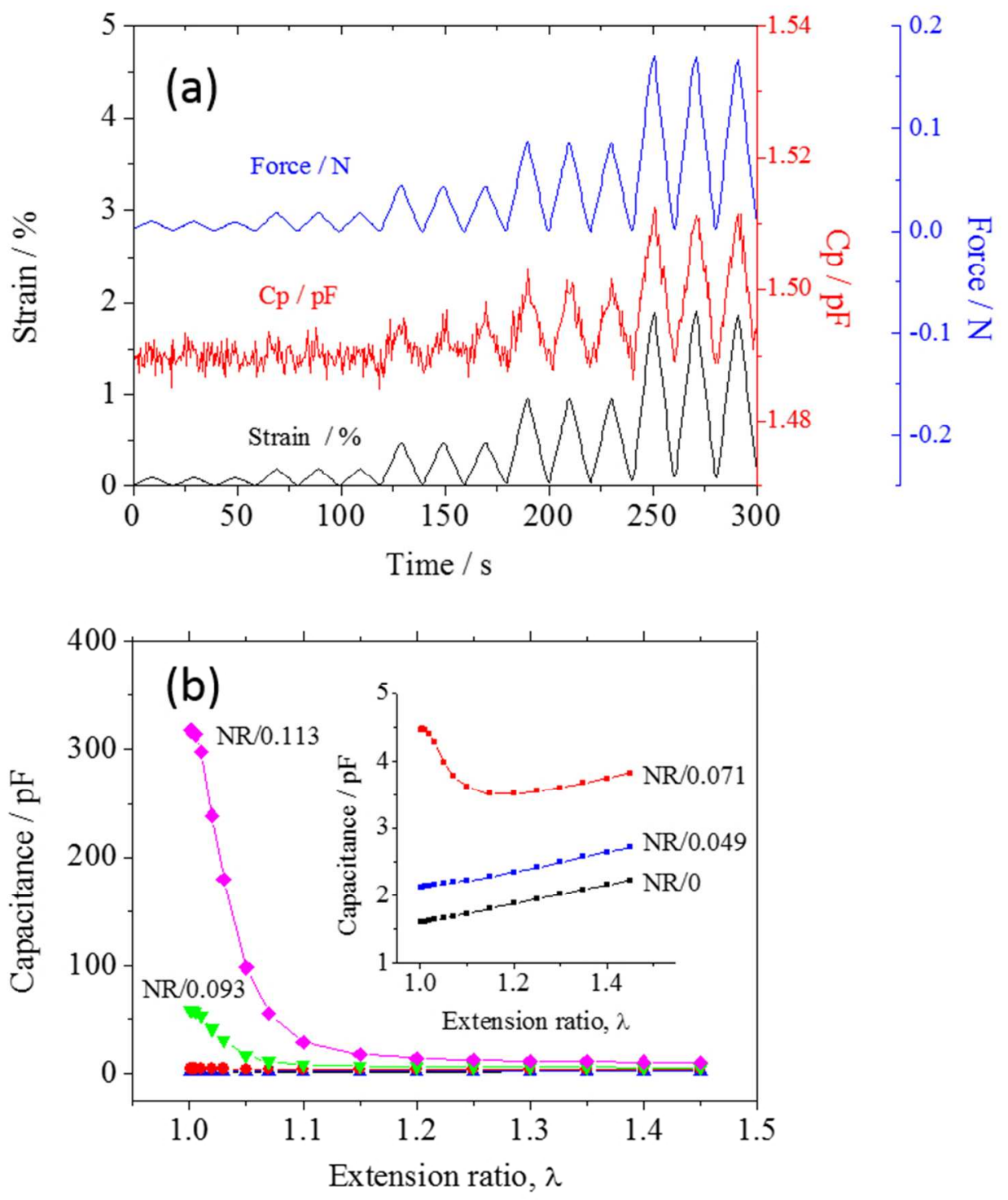

Figure 4. (a) Sensitivity limits of the SDMS method. The data is based on a single SDMS test of unfilled NR. All the dielectric data was measured at $1 \mathrm{kHz}$ electrical frequency. (b) Measured capacitance under strain for different samples. The inset graph shows a magnification of data for NR/0, NR/0.049 and NR/0.071. $80 \times 95 \mathrm{~mm}(249 \times 249 \mathrm{DPI})$ 


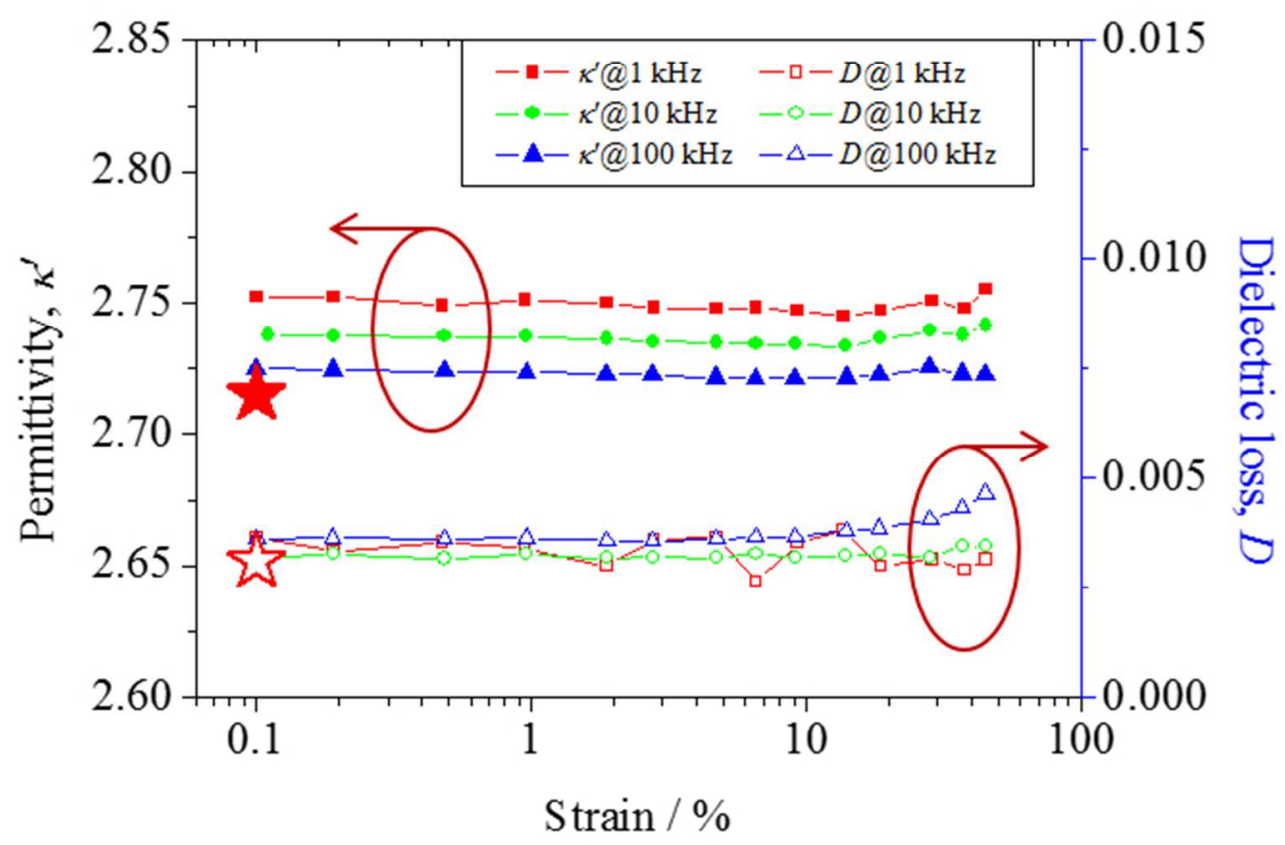

Figure 5. The dielectric behavior of unfilled NR as a function of strain. The filled red star is $\kappa^{\prime}$ measured by Novocontrol Concept 40 Dielectric Spectrometer at $1 \mathrm{kHz}$ and the hollow star is the corresponding dielectric loss data, D.

$80 \times 56 \mathrm{~mm}(214 \times 214 \mathrm{DPI})$ 


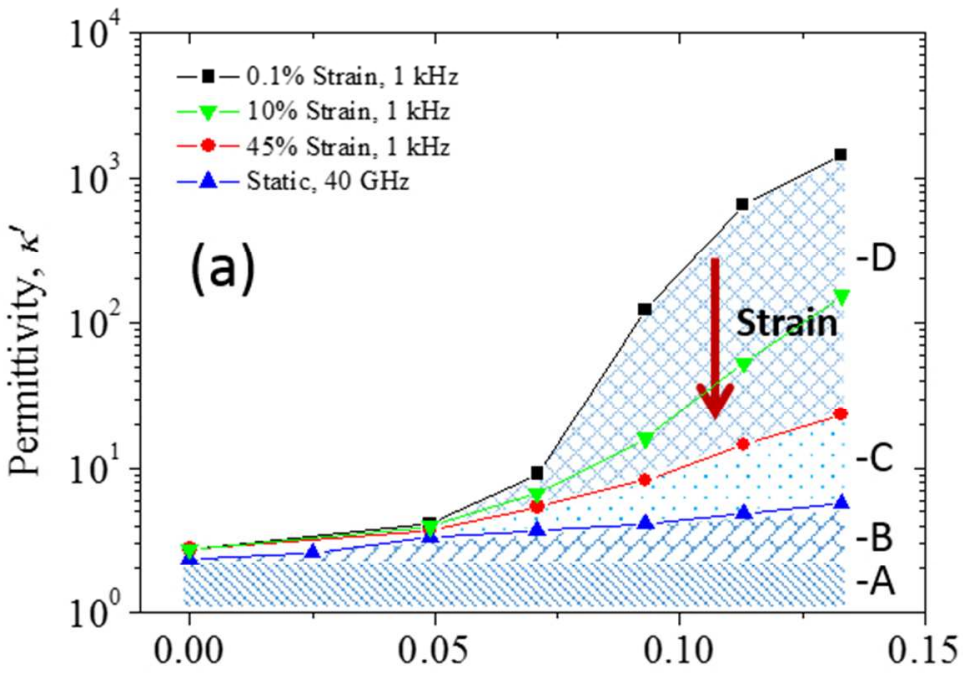

Filler volume fraction, $\phi$

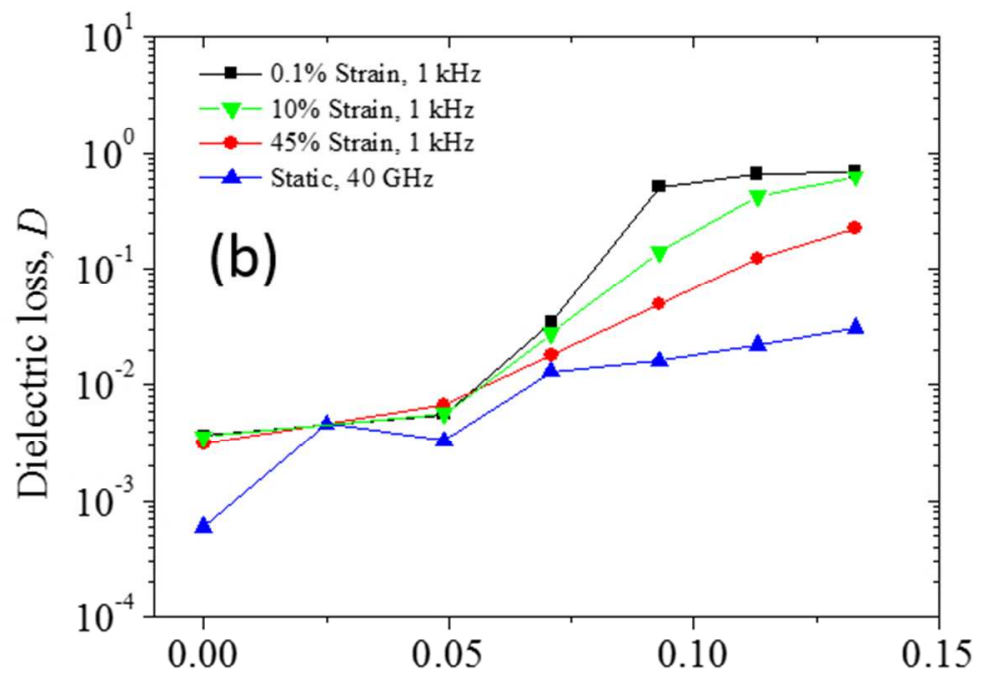

Filler volume fraction, $\phi$

Figure 6. (a) The relationship between the real part of permittivity $\kappa^{\prime}$ and filler volume fraction $\phi$ under different frequencies and strain conditions. (b) Dielectric loss against $\phi$ under different conditions. $80 \times 108 \mathrm{~mm}(216 \times 216 \mathrm{DPI})$ 

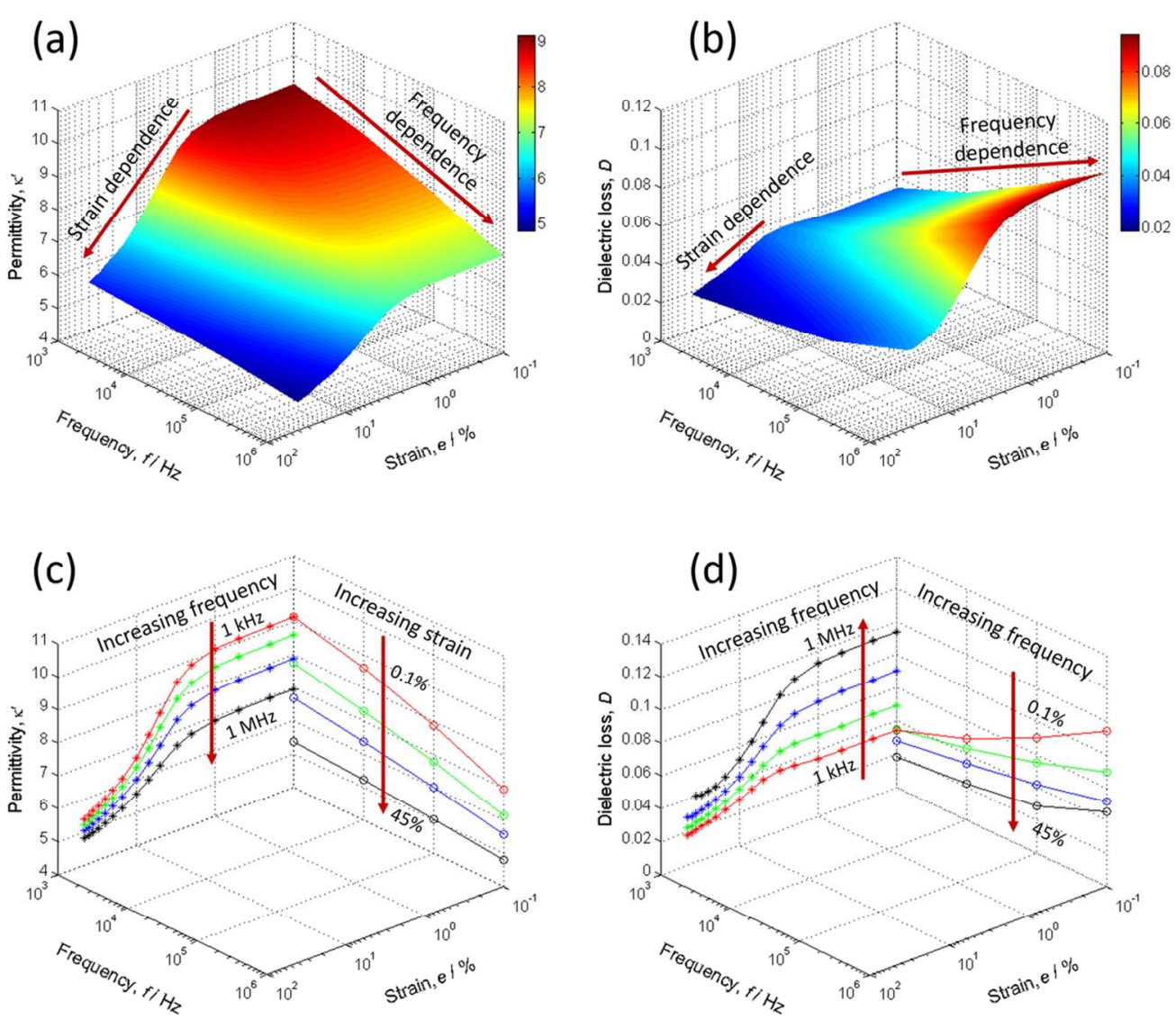

Figure 7. (a) \& (b) Strain e and electrical frequency $f$ dependent behavior of $\kappa^{\prime}$ and $D$ of NR/0.071. (c) \& (d) Projections of data from (a) and (b) respectively with selected data. Solid lines connect the data measured at the same strain (frequency- $\kappa^{\prime}$ plane) or frequency (strain- $\kappa^{\prime}$ plane) as guides for the eye. $107 \times 95 \mathrm{~mm}(300 \times 300$ DPI) 

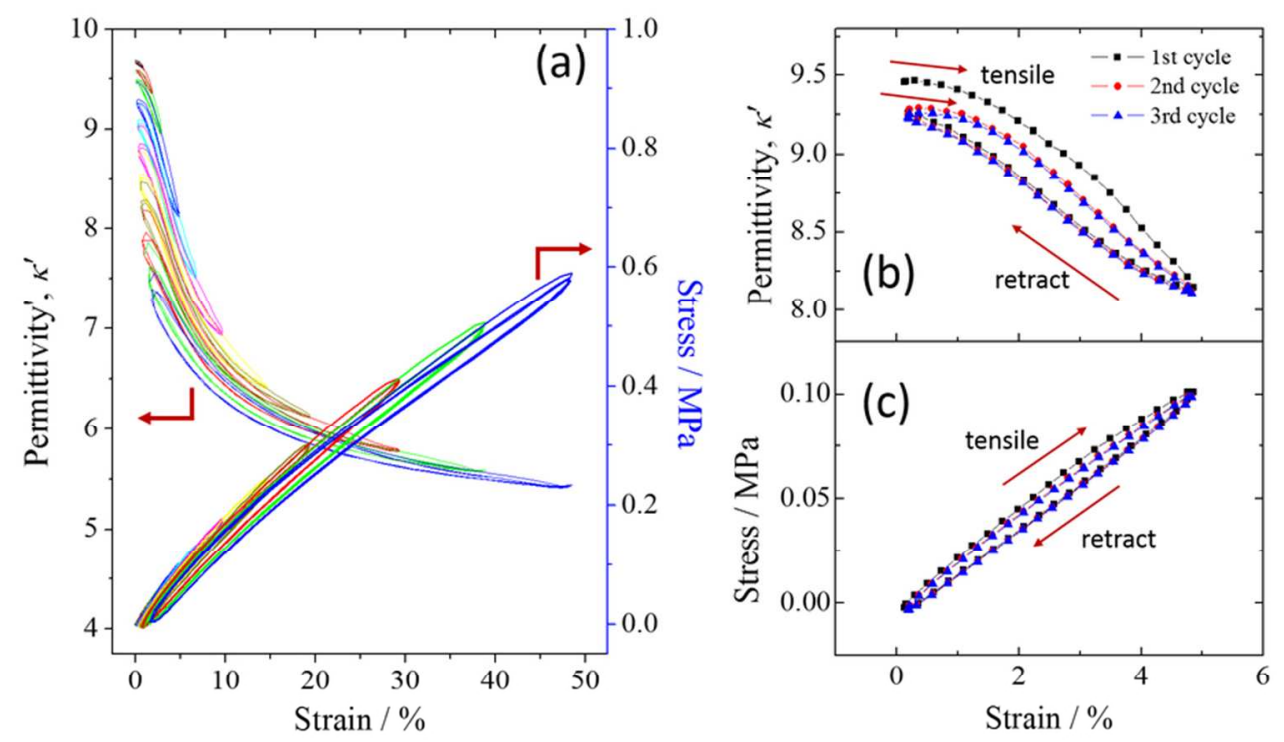

Figure 8. $K^{\prime}$ and stress vs strain for NR/0.071. (a) Multi-hysteresis data from 0 to $50 \%$ strain. (b) \& (c) Subdata taken from the multi-hysteresis sweeps showing the 0-5\% cycle data more clearly. All SDMS data was measured at an electrical frequency of $1 \mathrm{kHz}$. All data collected during a test is presented. $80 \times 53 \mathrm{~mm}(300 \times 300 \mathrm{DPI})$ 


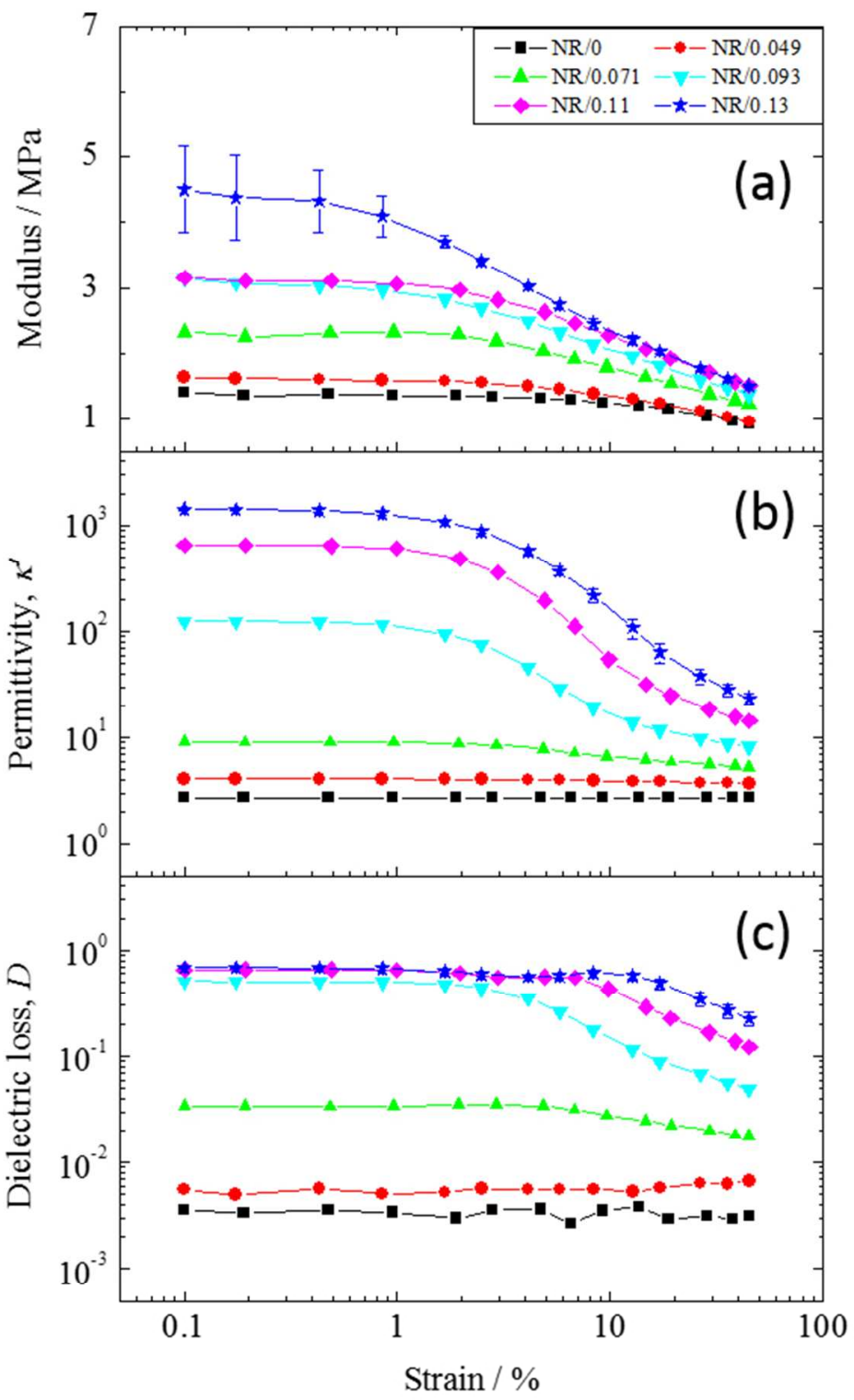

Figure 9. (a) Static modulus (stress/strain) vs strain collected simultaneously with the dielectric data. (b) \& (c) Strain-dependence of $K^{\prime}$ and $D$ of all samples (measured at an electrical frequency of $1 \mathrm{kHz}$ ). $80 \times 112 \mathrm{~mm}(215 \times 215 \mathrm{DPI})$ 